\title{
marges Marges
}

revue d'art contemporain Revue d'art contemporain

06 | 2007

Art et etnographie

\section{Art et ethnographie}

Art and Ethnography

\section{Claire Fagnart}

\section{OpenEdition}

\section{Journals}

Édition électronique

URL : http://journals.openedition.org/marges/829

DOI : $10.4000 /$ marges. 829

ISSN : 2416-8742

\section{Éditeur}

Presses universitaires de Vincennes

\section{Édition imprimée}

Date de publication : 15 octobre 2007

Pagination : 8-16

ISBN : 978-2-84292-250-4

ISSN : 1767-7114

Référence électronique

Claire Fagnart, "Art et ethnographie », Marges [En ligne], 06 | 2007, mis en ligne le 15 octobre 2008, consulté le 02 mai 2019. URL : http://journals.openedition.org/marges/829 ; DOI : 10.4000/ marges.829 


\section{Art et ethnographie}

1 Hal Foster, « L'artiste comme ethnographe ou la "fin de l'histoire" signifie-t-elle le retour de l'anthropologie ? ", dans Face à l'histoire, Paris, Centre Georges Pompidou, 1996, p. 498505.

${ }^{2}$ De l'ethnographie à l'ethnologie puis à l'anthropologie, il semble y avoir une intensification du caractère théorique de la recherche.

L'ethnographe constitue, à partir d'enquêtes de terrain, un fond documentaire ; l'ethnologue analyse et interprète ces documents, l'anthropologue théorise une conception générale
Dans son article " L'artiste comme ethnographe ${ }^{1}$ ", Hal Foster aborde la question des relations entre art et ethnographie ${ }^{2}$. L'auteur s'interroge sur les limites et la validité de ces échanges. Il pose aussi la question de la portée critique de cet « art ethnographique » qu'il considère comme un paradigme de l'art « de gauche ».

En guise d'introduction à cette journée d'études, j'essayerai de préciser les facteurs qui ont favorisé le développement des liens entre art et ethnographie. Parallèlement, j'évoquerai les interrogations de Hal Foster quant au potentiel critique de ces formes d'art. Enfin, pour terminer, je reposerai à ma manière la question de l'auteur.

Les liens entre l'art et l'ethnographie se sont plus particulièrement tissés à partir et autour d'une crise conjointe de l'anthropologie et de l'art. Nous examinerons pour commencer en quoi la crise de l'anthropologie a joué un rôle dans le développement de l'« art ethnographique ». Nous aborderons cette question à partir de deux entrées, l'une thématique, l'autre méthodologique³. Nous verrons ensuite en quoi la crise de l'art a pu favoriser le développement d'un « art ethnographique ». 
de l'homme, en relation avec sa culture. Les frontières entre ces trois champs d'étude sont donc aisées à traverser (un même individu peut être tour à tour ethnographe, ethnologue et anthropologue). L'expression « art ethnographique " s'applique à des pratiques artistiques de type documentaire plutôt qu'à des pratiques artistiques de type expressif.

${ }^{3}$ La question de la méthode concerne les modalités de déroulement des enquêtes de terrain observation ou participation - et, à la suite de l'enquête, la constitution d'un texte à visée scientifique rendant compte des données collectées lors de l'enquête ou l'écriture d'un texte «subjectif ».

4 Marc Augé, Non-lieux. Introduction à une anthropologie de la surmodernité, Paris, Seuil, 1992, p. 28.

${ }^{5}$ Et l'on pourrait affirmer que cette question est également celle de la philosophie. Mais, alors que l'anthropologie aborde cette question en relation avec le contexte culturel, ce n'est pas le cas de la philosophie. (Cf. Marie GailleNikodimov, « Comment réduire l'altérité ? L'anthropologie et la philosophie face à l'idée de contexte culturel », dans Critique $\mathrm{n}^{\circ}$ 680-681, "Frontières de l'anthropologie », 
janvier-février 2004).

${ }^{6}$ Cette conception téléologique de l'anthropologie, qui repose sur une croyance dans le progrès, fut au $19^{\mathrm{e}}$ siècle celle de Comte, Tylor ou Frazer.

${ }^{7}$ Au culturalisme américain, on peut aujourd'hui opposer le cognitivisme qui cherche à décrire ce que pensent les individus, plutôt que leurs manières d'agir. Le cognitivisme est donc une anthropologie de la pensée plutôt que des comportements. Il a la prétention d'élaborer un modèle théorique universel du

fonctionnement de la pensée humaine. Il s'agit en ce sens d'une approche qui favorise un modèle universaliste et rejoint pour cette raison l'anthropologie traditionnelle du $19^{\mathrm{e}}$ siècle.

8 Marc Augé, Le Sens des autres, Paris, Fayard, 1994, p. 10.

9 On constate que cette situation, qui devrait théoriquement pouvoir favoriser un enrichissement des identités et des cultures et un partage des territoires, induit, dans de nombreux cas, ghettoïsation, déficit symbolique, effacement des cultures, raidissement des identités. Comme si l'autre, plus proche, était beaucoup plus inquiétant. La mondialisation peut s'accompagner d'un caractéristique essentielle de notre actualité ${ }^{\mathbf{8}}$ ». L'anthropologie est devenue non exotique. De l'étude des peuples lointains, elle est passée à l'étude de milieux lointains, comme par exemple la paysannerie. Aujourd'hui, l'ethnologie ne se consacre plus à l'étude exclusive de petits groupes « ethniques » plus ou moins menacés de disparition. On enquête dans les prisons, les hôpitaux, les écoles, chez les SDF, les malades atteints du SIDA... L'autre aujourd'hui c'est le prolétaire, c'est l'immigré. L'altérité sociale et culturelle est devenue interne et non plus externe. Les grandes villes, espaces de mondialisation, sont devenues des lieux de rencontre de cultures. En ce sens, la fin de l'exotisme ouvre sur un champ d'exploration complexe qui est celui des identités plurielles et du pluralisme culturel ${ }^{9}$. Les frontières se sont déplacées : de territoriales, elles sont devenues économiques. Elles séparent ceux qui sont dans le système et ceux qui sont en-dehors, à l'intérieur d'un même espace géographique.

Quand nous parlons d'art ethnographique, c'est bien de cet autrelà qu'il s'agit. Si cette nouvelle altérité peut, plus que l'autre exotique, faire l'objet d'une thématique artistique, cela peut sans doute s'expliquer par le fait très pragmatique qu'elle ne nécessite pas que l'artiste bénéficie d'un soutien financier pour pouvoir s'en approcher.

D'autre part, on peut noter, à la suite de Hal Foster, que l'oppression raciale ou néo-colonialiste se superpose à l'oppression capitaliste de classe telle qu'on la désignait au début du $20^{\mathrm{e}}$ siècle et, qu'en ce sens, le paradigme de "l'artiste comme ethnographe » n'a pas vraiment valeur de nouveauté. L'autre culturel d'aujourd'hui n'est pas différent de l'autre social d'hier ; l'artiste comme ethnographe reprend à son compte la volonté critique qu'eut avant lui l'artiste producteur du constructivisme russe par exemple. Mais « l'artiste comme producteur » s'inscrivait dans une perspective utopique selon laquelle l'art aurait le pouvoir de changer la réalité. " L'artiste comme ethnographe " s’inscrit dans une perspective non utopique, réflexive et non transitive selon laquelle il n'y a pas de lien direct entre art (esthétique) et réalisation politique. L'art ethnographique ne prétend pas à une transformation du monde. L'artiste est une courroie de transmission entre des milieux, des cultures différentes. Hal Foster se demande aussi s’il y a, dans cette thématique, idéalisation ou mythification de l'autre comme un « être authentique ${ }^{10}$ ». Malgré toutes ces remarques, nous retiendrons la dénomination "d'art ethnographique » pour désigner un art à visée documentaire et réflexive, consacré à l'autre considéré dans le cadre de sa culture, sans ambition utopique. 
phénomène de déculturation ou d'acculturation qui nous concerne directement et qui constitue un nouvel objet - largement plus complexe-de l'anthropologie. concourent au resserrement des liens entre art et ethnographie.

$\mathrm{Au}$ lieu de considérer l'ethnologie à partir de son objet ${ }^{\mathbf{1 2}}$, nous pourrions avancer l'idée qu'elle se conçoit à partir de sa méthode ${ }^{13}$. L'enquête de terrain, source principale de sa légitimité, en définirait la spécificité14. Plutôt que chercher à « comprendre » l'autre dans le cadre de sa culture, il s'agit de réfléchir aux caractéristiques des échanges qu'il noue ; plutôt que de poser la question de l'autre, il s'agit de poser la question de ses relations. Mais alors l'ethnologue est immédiatement renvoyé à ses relations avec son interlocuteur et, ce faisant, renvoyé aux modalités concrètes d'enquête. Ici, l'ethnologie est définie comme étude empirique, de terrain, sans préjugés. Comme nous l'avons dit plus haut, traditionnellement la discipline s'est développée à partir de séjours d'Occidentaux dans divers cadres exotiques sans que l'avis des personnes accueillantes ne soit sollicité. Mais la conscience de la complexité des relations entre les enquêteurs et leurs interlocuteurs s'est affinée avec le temps. D'abord toute d'extériorité et d'observation, elle a pu se faire négociation, dialogue, participation, etc. Conjointement s'est développée la conscience que l'anthropologue ne pouvait prétendre à accéder à la vérité du sujet parlant, ou encore ne pouvait prétendre le comprendre de l'intérieur. C'est à partir des années 1970 que s'est réalisé cet examen autocritique de l'anthropologie.

Parallèlement, une réflexion sur la restitution écrite des données collectées pendant l'enquête s'est développée. Cette réflexion a particulièrement développé deux groupes de questions. Les unes concernent le dilemne description/interprétation : le discours structuraliste qui traite la culture comme un texte pouvant être lu, analysé, interprété, déchiffré. Les autres questions concernent la manifestation, dans le récit anthropologique, des subjectivités à l'œuvre pendant l'enquête : le discours poststructuraliste qui cherche à engager un dialogue avec l'autre.

\section{La question des subjectivités}

Jusque dans les années 1980, les textes d'anthropologie s'inscrivaient dans le registre stylistique du « réalisme positiviste »

\section{$\mathbf{1 0}^{\mathbf{H}}$ Hal Foster met en garde contre cette idéalisation/mythification (excès d'identification) qui enferme l'autre " dans une hiérarchie de douleur qui implique que les victimes ne puissent mal agir » (ibid., p. 505). \\ ${ }^{11}$ Certains théoriciens comme Clifford ou Le Caisne ont récemment proposé une description précise des modalités de déroulement des enquêtes ethnographiques.}

12 C'est la démarche de Marc Augé à propos de l'anthropologie.

${ }^{\mathbf{1 3}}$ Les principales méthodes de l'ethnologie sont l'usage de la photographie, le recueil de rites, la description des lieux, les entretiens avec les observateurs tandis que les méthodes propres à la sociologie sont les questionnaires et les statistiques. Il est évidemment tentant, après Durkheim ou Bourdieu, de s'opposer à ces divisions disciplinaires qui tendent à figer les pratiques alors qu'elles se trouvent au service d'un même type d'interrogations.

\footnotetext{
14 Pour cette partie, nous nous référons à JeanPaul Colleyn,
} 
« L'ethnographie, essais d'écriture ", dans Critique $\mathrm{n}^{\circ}$ 680-681, op. cit., p. 139-149.

15 Jean-Paul Colleyn, ibid., p. 142.

${ }^{16}$ À ce sujet, voir le très riche - et très discutable - ouvrage de James Clifford et George Markus qui rend compte d'un séminaire ayant eu lieu en avril 1984 à Santa Fe (School of American Research) sur la question de la production de l'écrit ethnographique : James Clifford et George Marcus, Writing Culture, the Poetics and Politics of Ethnography, Berkeley, University of California Press, 1986.

${ }^{\mathbf{1 8}}$ Jean-Paul Colleyn, art. cit., p. 142.

19 Marc Augé, Un Ethnologue dans le métro, Paris, Hachette Littératures, 1986.

${ }^{20}$ Michael Taussig, The Magic of the State, New York, Routledge, 1997. issu du $19^{\mathrm{e}}$ siècle $^{15}$. Le " je » et le « tu » étaient bannis en tant que traces subjectives de l'intervention de l'anthropologue alors même que l'enquête était fondée sur une relation personnelle entre le " scientifique » et " l'interlocuteur ». L'anthropologie américaine a remis en question ces conventions narratives, engageant une rupture avec les « conventions d'un réalisme sans point de vue $\mathrm{e}^{16}$ ». Il est alors apparu comme une évidence que le texte était dépendant du type de relation que l'enquêteur noue avec ses interlocuteurs, de la personnalité des uns et des autres, que l'enquête était avant tout une expérience de communication et que la transposition écrite d'une expérience de terrain ne pouvait pas être objective ${ }^{17}$. Dès lors, « un programme s'est imposé : repartir du sujet ${ }^{\mathbf{1 8}}$ ». Parallèlement, une critique $\mathrm{du}$ « monologisme » a été faite et un « dialogisme » a été autorisé, selon lequel l'anthropologue assume - et donc rend présent dans son texte - le dialogue qu'il a noué avec ses interlocuteurs. Cette conscience nouvelle du caractère intersubjectif du travail ethnographique a poussé les anthropologues à rompre avec l'écriture universitaire. On peut penser ici au Marc Augé de Un Ethnologue dans le métro ${ }^{99}$ ou à Michael Taussig ${ }^{20}$. Cette tendance à la subjectivation de l'écrit ethnologique conduit à rendre perméables, de façon discutable, les frontières entre écriture scientifique et artistique.

D'autre part, le dialogisme - correspondant à une reconnaissance de l'apport de l'interlocuteur à l'enquête - se retrouve en des termes assez proches dans la culture artistique de la participation. Il s'agit alors pour l'artiste d'affirmer la position de sujet des individus qu'il représente afin d'éviter à tout prix que l'œuvre ne les réifie. « L'artiste comme ethnographe » cherche à faire participer l'autre pour éviter sa réification et pour que son travail gagne en efficacité politique. Dans les séries de portraits réalisés avec des exclus, des lycéens de La Seine Saint-Denis ou encore des Compagnons d'Emmaüs, Marc Pataut cherche à rompre avec l'extériorité du photographe au profit d'une quête d'empathie. Il élabore des procédures spécifiques permettant à ses modèles de se faire sujets, c'est-à-dire partenaires actifs du processus photographique. On peut aussi évoquer Sylvie Blocher qui dans Are You a Masterpiece ? cherche à établir un dialogue avec ses modèles ; Gillian Wearing qui dans Signs That Say What You Want Them to Say and not Signs That Say What Someone Else Wants You to Say (1992-93) tend à des passants anonymes papiers et marqueurs, les enjoignant de noter leurs sentiments ou opinions du moment. Enfin, je voudrais évoquer les photographies qu'Olivier Pasquier a réalisées dans un lieu d'accueil de SDF dit « la Moquette ». Il y a eu 
ici un travail de revalorisation de ces gens par l'image et par le texte (réalisé en atelier d'écriture et répondant à la question « qui êtesvous ? »). Le travail n'a pas tant consisté à rendre visible le réel mais à fabuler ensemble : jouer à sortir de l'impasse laisse entrevoir qu'il est possible de s'en sortir. Dans tous ces cas, le chemin vers l'autre consiste à lui donner une place active.

On peut se demander quelle conscience ont les artistes de ce modèle anthropologique tant sur le plan thématique que méthodologique. Hal Foster s'interroge vivement sur la méthode « dialogique » empruntée à l'anthropologie. Prenant pour exemple le «Projet Unité » (1993, réhabilitation de logements sociaux de Le Corbusier), où la population, largement immigrée, était invitée à s'auto-représenter - les documents proposés (cassettes audio, photos de famille) furent considérés et exposés comme des documents anthropologiques - Hal Foster note que, outre leur condescendance sociale, les artistes ne s'interrogèrent pas sur leur " autorité ethnographique ${ }^{21}$ » ni sur cet emprunt direct à l'anthropologie dialogique.

\section{Le dilemme description/interprétation}

Wittgenstein, dans le Rameau d'or de Frazer, semble être le premier à avoir fait une critique de l'anthropologie explicative, linéaire, rationaliste et herméneutique. Sa critique porte sur l'anthropologie en tant que science explicative dont le modèle est l'herméneutique textuelle. Il recommande alors de se limiter à une approche exclusivement descriptive.

Bourdieu ${ }^{22}$ et Bloch ${ }^{23}$ se sont interrogés sur « les modèles à travers lesquels nous pensons la pensée des autres, selon une démarche réflexive qui s'interroge sur ses propres catégories de pensée avant de prétendre décrire celle des autres, faute de laquelle on risque toujours de projeter les premières sur les secondes ${ }^{24} »$. Ils ont donc mis l'accent sur le fait que nous percevons la vie des autres « à travers nos propres catégories de jugement ${ }^{25}$ ».

Clifford et Marcus ont élaboré une critique de la prétention scientifique à l'objectivité de l'orientation culturaliste (relativiste) de Boas et Sahlins. Dans le livre cité plus haut, ces auteurs mettent en évidence que l'anthropologie relativiste omet le caractère perspectiviste de ses analyses. En voulant expliquer l'autre par sa culture exclusivement, les anthropologues culturalistes tendent à oublier qu'ils font usage dans leurs explications de leurs propres catégories de pensées, de leur propre culture, et ce faisant
21 Hal Foster, « L'artiste comme ethnographe... », art. cit., p. 503.

22 Pierre Bourdieu, Le sens pratique, Paris, Minuit, 1980.

${ }^{23}$ Maurice Bloch, How We Think They Think: Anthropological Approaches to Cognition, Memory and Literacy, Oxford/Boulder, Westview Press, 1998. Ce livre est une réponse à Lucien. Lévy-Bruhl, How Natives Think: traduction anglaise de Bunzel du livre de LévyBruhl, Les Fonctions mentales dans les sociétés inférieures, Paris, Alcan, 1910 et à Marshall Sahlins, How "Natives" Think: About Captain Cook for Example, Chicago/Londres, The University of Chicago Press, 1995.

24 Frédéric Keck, « Une Ethnographie de l'esprit est-elle possible ", dans Critique $\mathrm{n}^{\circ} 680$-681, art. cit., p. 95-96.

25 Marie GailleNikodimov, art. cit., p. 125. 
${ }^{26}$ Frédéric Keck, art. cit., p. 96.

${ }^{27}$ Hal Foster, art. cit., p.501. produisent une interprétation et non une explication. Pour ces auteurs, le culturalisme correspond à une herméneutique. Mais plutôt que de se limiter à une approche descriptive, Clifford et Marcus recommandent que l'anthropologie - y compris culturaliste - renonce à toute prétention à l'objectivité. Pour ces auteurs, « il n'y a que des points de vue et des interprétations en sorte que l'anthropologie doit renoncer à sa prétention scientifique pour devenir une forme d'écriture poétique et littéraire ${ }^{26}$ ». À noter que pour Hal Foster, cette figure de l'anthropologue devenu interprète artistique du texte culturel est un malentendu.

Cette conscience toute nietzschéenne du " perspectivisme » de l'anthropologie a donc conduit à deux réponses antagonistes : l'une exclusivement descriptive, l'autre radicalement interprétative. II semble ne pas y avoir de position intermédiaire, à moins que l'anthropologue ne soit confronté à un monde qui lui est familier et qu'il peut comprendre. En effet, si le monde auquel il est confronté lui est étranger, il ne peut que l'observer comme on observe un jeu dont on ne connaît pas les règles, ou alors l'interpréter. Et l'interprétation est une fiction dans la mesure où elle est une représentation que se fait l'anthropologue de la culture de l'autre.

On entend bien ici que cette autocritique de l'anthropologie - et particulièrement la réponse interprétative - a ouvert des brèches, des passages entre l'art et l'ethnographie, a permis des recouvrements de pratiques, autorisant l'anthropologue à se faire " artiste » ou poète et du même coup favorisant l'appropriation de la pratique ethnographique par les artistes. Là encore, Hal Foster s'interroge sur la validité du travail d'artistes qui se prennent pour des ethnologues, aspirant à " une vie d'enquêteur de terrain ", s'inspirant souvent indirectement « des principes fondamentaux de la tradition de l'observateur participant ${ }^{27}$ ». Se pose aussi la question d'un malentendu plutôt que d'un échange entre art et anthropologie. Les méthodes de l'anthropologie sont-elles vraiment exportable dans le champ de l'art?

\section{Crise de l'art}

Si l'on peut envisager le développement de liens entre l'art et l'anthropologie à partir d'une crise de l'anthropologie, on peut aussi appréhender la question à partir d'une crise de l'art. Dans ce contexte, je voudrais évoquer le fait que le resserrement des liens entre art et ethnographie peut aussi être perçu comme la confirmation supplémentaire de l'émergence d'une nouvelle figure 
de l'artiste, dans le contexte « postmoderne » (ou "surmoderne ») de la déconstruction du sujet.

Nous savons qu'à l'art traditionnel comme production intentionnelle d'un objet fini de contemplation et d'interprétation correspondent deux figures principales de l'artiste : la première est celle de la conception classique de l'art, selon laquelle l'artiste parvient à exprimer une vérité partagée de tous, il est un découvreur d'une vérité préexistante ; la seconde fait de l'artiste l'inventeur d'un monde. Dans les deux cas, l'artiste est un sujet fort, un auteur.

La figure de l'artiste qui se développe avec notre société actuelle est plutôt celle d'un intermédiaire. On retrouve ici une figure déjà présente au Moyen-Âge ou dans les sociétés théocratiques qui n'ont pas développé de philosophie du sujet. Dans les sociétés théocratiques, l'artiste est généralement un médiateur entre les forces divines et les spectateurs. La figure de l'artiste comme passeur, rejaillie au $20^{\mathrm{e}}$ siècle, le met dans une multiplicité de situation de médiation. Deux exemples parmi plusieurs : avec le Land art, il est médiateur entre la nature et le spectateur ; avec l'art informel, il est un médiateur entre la matière elle-même, sa force expressive et le spectateur. Cette position de l'artiste comme médiateur (intermédiaire, médium) peut directement être rapportée à l'affaiblissement du sujet philosophique propre à notre société surmoderne.

L'artiste comme ethnographe se positionne lui aussi comme médiateur, intermédiaire entre "l'autre » et les spectateurs. C'est en ce sens que la crise de l'art - qui est plutôt redéfinition de l'art et du travail artistique dans la société technologique - pousse au développement de cette figure de l'artiste comme ethnographe. II se retrouve alors muni des instruments technologiques d'enquête (enregistreurs de son et/ou d'images) comme un médiateur (un intermédiaire, un médium) donnant une visibilité à des individus généralement condamnés à l'invisibilité (les insoumis, les rejetés du système, les exclus, etc.). Comme le dit André Rouillé à plusieurs reprises dans son livre sur la photographie, il s'agit de rendre le réel visible et non de le représenter, de donner une visibilité au réel en évitant qu'il ne se détruise en se montrant. Là encore, l'instauration, au cœur du processus photographique, du dialogue entre le photographiant et le photographié, permet d'éviter que l'image ne soit un « objet inerte à contempler ${ }^{28}$ ». Réalisée par ceux qu'elle montre, elle peut ainsi éviter l'esthétisation, la marchandisation, la transformation de leur marginalité en objet d'art. 
${ }^{29}$ Clifford Geertz, The Interpretation of Cultures, New-York, Basic Books, 1973.

${ }^{30}$ Roy Dilley, The Problem of Context, Oxford/New York, Berghahn Books, 1999.

${ }^{31}$ Marie GailleNikodimov, art. cit., p. 119.

32 Ibid.

\section{À propos du pouvoir en guise de conclusion}

Je reviendrai pour conclure sur la question du pouvoir de l'artiste comme de l'anthropologue. Nous avons vu plus haut que Clifford et Marcus pensent l'ethnographie comme une activité d'interprétation. C'est à Geertz ${ }^{29}$ que l'on doit l'idée que l'anthropologie n'est pas seulement interprétation d'une culture mais aussi interprétation d'une interprétation : celle que proposent les interlocuteurs (indigènes) dans l'enquête elle-même. Dilley $3^{30}$ souligne quant à lui le fait que l'interprétation doit tenir compte des enjeux de pouvoir mis en œuvre par la situation d'enquête. Ce dernier souligne que l'anthropologue qui s'affirme comme « interprète des cultures » s'appuie sur et perpétue une situation d'inégalité : il omet que la textualisation de la réalité - le récit qui met en lumière les éléments culturels " qui motivent et rendent compte des actes observés ${ }^{31} "$ - n'est pas l'apanage de l'anthropologue. L'interlocuteur participe aussi à cette textualisation. Reconnaître cette dette implique que le statut d'auteur soit partagé par l'enquêteur et ses interlocuteurs même s'il s'agit d'un partage inégal dans la mesure où les interlocuteurs restent soumis au projet de l'anthropologue. Par ailleurs, les interprétations ne sont pas généralement destinées aux interlocuteurs mais aux collègues. L'échange herméneutique est donc inégal. "Enfin, le caractère impérialiste de l'interprétation anthropologique est d'autant plus affirmé lorsque l'anthropologue s'attache à l'étude d'un espace ou d'une question qui a déjà fait l'objet d'enquête avant la sienne ${ }^{32}$ ». Les interprétations sont alors enfermées dans des débats internes à l'anthropologie.

Hal Foster reprend cette critique et l'applique à l'art ethnographique. Il constate que, s'il arrive que les autorités sociales ou politiques soient contestées dans l'art ethnographique, l'autorité de l'artiste ne l'est jamais, même quand l'artiste développe une esthétique dialogique et participative.

\section{Claire Fagnart}

